\title{
Quasistatic response of loose cohesive granular materials
}

\author{
Walid Lammali ${ }^{1, *}$, Jean-Noël Roux ${ }^{1, * *}$, and Anh-Minh Tang ${ }^{1, * * *}$ \\ ${ }^{1}$ Lab Navier, Univ Gustave Eiffel, École des Ponts, CNRS, 14-20 boulevard Newton, 77427 Champs-sur-Marne, France
}

\begin{abstract}
DEM-simulated model cohesive assemblies of spherical grains of diameter $d$, with contact tensile strength $F_{0}$, once prepared in loose states, are quasistatically subjected to growing isotropic pressure $P$, and then to triaxial compression, maintaining lateral stresses $\sigma_{2}=\sigma_{3}=P$ while increasing axial stress $\sigma_{1}=P+q$ and strain $\epsilon_{1}$. Reduced pressure $P^{*}=d^{2} P / F_{0}$ varies from 0.1 (cohesion dominated case, for which systems typically equilibrate with solid fraction $\Phi \simeq 0.35$ ), to large values for which the cohesionless behavior is retrieved. In triaxial compression, while the moderate strain response $\left(\epsilon_{1} \sim 0.1\right)$ is influenced by initial coordination numbers and mesoscale heterogeneities, the approach to the critical state, as both $q$ (deviator) and $\Phi$ steadily increase, gets slower for smaller $P^{*}$. Critical ratio $q / P$ strongly increases for decreasing $P^{*}$, as roughly predicted in an "effective stress" scheme. Anomalously small elastic moduli are observed in the gel-like structures. While extensive geometric rearrangements take place, no shear banding is observed. Loose cohesive granular assemblies are thus capable of large quasistatic stable plastic strains and ductile rupture.
\end{abstract}

\section{Introduction}

Numerical grain-level investigations, by discrete element methods (DEM)[1], of the properties of assemblies of hard objects interacting by frictional contacts [2] are now quite widespread, and their quasistatic mechanical behavior, as probed, e.g., by the standard triaxial test used in geotechnique labs with sands [3], is often addressed. Cohesive granular assemblies, such as powders and colloids, are less frequently studied, but exhibit a wider variety of static states. While the solid fraction $\Phi$ of cohesionless assemblies of spherical identical beads ranges from the random close packing value $\simeq 0.64$ down to some frictiondependent minimum, in the $0.55-0.6$ range, much looser static cohesive assemblies may be observed [4], in which applied stresses are carried by tenuous, ramified contact networks reminiscent of colloidal gels [5, 6].

The present contribution reports on a DEM investigation of the behavior of a simple model material, the properties of which are briefly recalled in Sec. 2, prepared in $P^{*}$-dependent states by isotropic compression (Sec 3), and subjected to triaxial compression (Sec 4). A quick final discussion (Sec 5) suggests perspectives.

\section{Simulated model}

\subsection{Particle interactions}

We use the same model system as in Refs. [4, 7]: spherical beads of diameter $d$ and mass $m$, with Hertz contact elasticity, friction coefficient $\mu=0.3$, and adhesive forces chosen according to a simplified model of capillary attraction

\footnotetext{
*e-mail: walid.lammali@univ-eiffel.fr

**e-mail: jean-noel.roux@univ-eiffel.fr

***e-mail: anh-minh.tang@enpc.fr
}

A video is available at https://doi.org/10.48448/vygx-k464 through small liquid bridges, which form in wet granular assemblies at low saturation. This results in an attractive contact force $F_{0}=\pi \Gamma d$ ( $\Gamma$ denoting the interfacial tension of the wetting liquid). One specific feature of this force is its hysteresis: liquid bridges formed at intergranular contacts survive between receding pairs, as long as the separation distance $D$ does not exceed a rupture threshold $D_{R}$ (here, $D_{R}=d / 10$, unless specified otherwise, corresponding to a meniscus volume $10^{-3} d^{3}$ ). One should distinguish the contact coordination contribution, $z_{c}$, and the distant interactions one, $z_{d}$, to the total coordination number $z=z_{c}+z_{d}$. The attractive force decrease as $D$ grows from zero to $D_{R}$ is modeled with a simple law [4, 7] ("Maugis approximation"). The Coulomb inequality involves the elastic repulsive normal force $F_{N}^{e}$ only. Thus, a contact with vanishing normal force $\left(F_{N}^{e}-F_{0}=0\right)$ may transmit a tangential force as large as $\mu F_{0}$. Remarkably, this simple numerical model agrees quantitatively with experimental observations in simple shear flow $[8,9]$ and in isotropic compression [4]. We use the same dimensionless control parameter as in Refs. [4, 8, 9], reduced pressure $P^{*}=d^{2} P / F_{0}$, comparing the adhesive force to pressure $P$. Cohesive effects are strong for small $P^{*}$, stabilizing tenuous, open structures [4]. Their influence gradually vanish as $P^{*}$ increases, until the properties of cohesionless systems are retrieved for $P^{*} \gg 1[4,10]$.

\subsection{Sample preparation}

Our DEM procedure involve several 8000 particle samples for each set of parameters, all enclosed in boxes with fully periodic boundary conditions. To compress samples to large enough 'axial' strains $\epsilon_{1}$ (counted positively) in triaxial tests, tall rectangular parallelipipedic samples are 
prepared, with aspect ratio 2 . Loose systems need to form aggregated structures to support stresses $[4,6]$. Our aggregation procedure is ballistic: grains, randomly distributed within the simulation cell, at solid fraction $\Phi=0.3$, are attributed Maxwell-distributed random velocities, and left to collide and interact until a unique, equilibrated cluster of aggregated grains spans the system. The initial mean quadratic velocity determines the final connectivity. $F_{1}=\max \left(d^{2} P, F_{0}\right)$ defining a relevant force scale, our equilibrium condition requests forces and torques to balance within respective tolerances $10^{-4} F_{1}$ and $10^{-4} F_{1} d$.

\section{Isotropic compression}

Once the initial aggregated state is achieved, the sample is subjected to stepwise increasing isotropic pressure values, and equilibrated at different $P^{*}$ levels The resulting com-

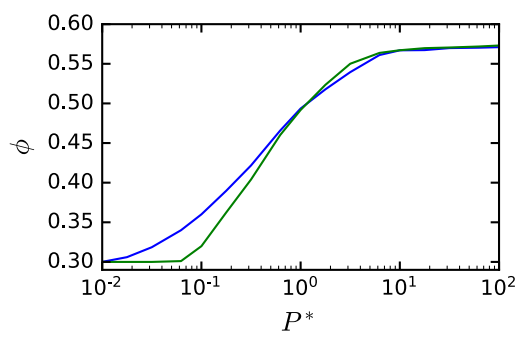

Figure 1. Isotropic compression curve for initial low (blue) and larger (green) coordination number (obtained with small or large initial kinetic energy in aggregation process).

pression curve (Fig. 1) is found independent of the details of the procedure, but remains sensitive to the initial solid fraction $\Phi_{0}$, and to the initial connectivity (see also [4]). Fig. 2 shows how better coordinated systems (right im-
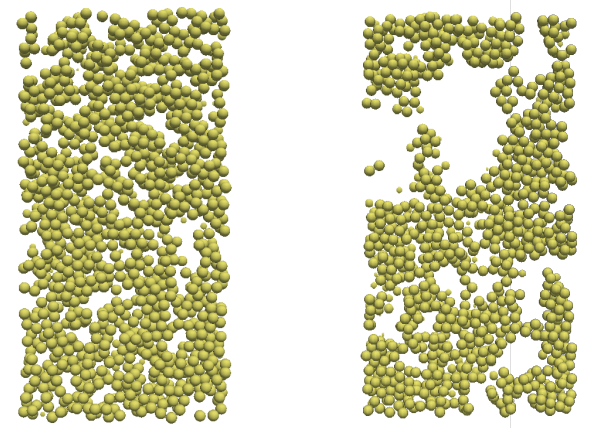

Figure 2. Views of slices (thickness $3 d$ ) cut through simulated systems compressed to $P^{*}=0.1$ (near initial states of compression in Fig. 1). Left: $\Phi=0.359, z_{c}=4.50, z_{d} \simeq 0.05$. Right: $\Phi=0.319, z_{c}=4.73, z_{d}=0.29$.

age), obtained with a stronger agitation in the initial aggregation stage (i.e., larger initial kinetic energy per grain, compared to $F_{0} D_{r}[4,6]$ ), comprise larger holes and dense regions. (The contact network only seems disconnected because a slab of thickness $3 d$ is shown). The Delaunay tesselation of the set of grain centres enables the partition of the void space into disjoint pores. The decrease of pore size in the compression is shown in Fig 3, which quantifies the enduring effects of the initial aggregation-induced geometric difference between the states depicted in Fig. 2.

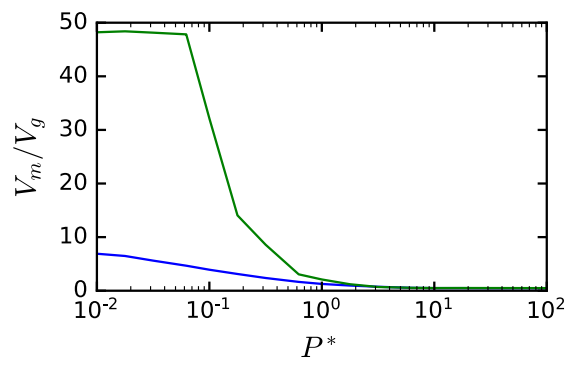

Figure 3. Volume-averaged pore volume $V_{m}$, normalised by grain volume $V_{g}$, versus $P^{*}$ in isotropic compressions of Fig. 1 (same colour code).

Such tenuous force-carrying structures have anomalous elastic moduli, expressing their response to small stress increments without network rearrangement or irreversible sliding in contacts. This is shown in Fig. 4, in which bulk $(B)$ and shear $(G)$ moduli, computed with

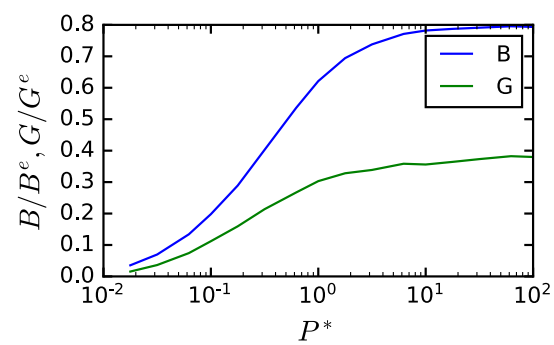

Figure 4. Normalised elastic moduli, $B / B^{e}$ and $G / G^{e}$, versus $P^{*}$, in compression of low-coordination system of Fig. 2.

the matrix method of Refs. [10, 11], are normalised by reference values $B^{e}, G^{e}$. Those are Voigt (homogeneous strain) estimates of the moduli, and their values, for HertzMindlin and a material with Young modulus $E$ and Poisson ratio $v$, read

$$
B^{e}=\frac{P_{e}^{1 / 3}}{2}\left[\frac{z_{c} \Phi E}{3 \pi\left(1-v^{2}\right)}\right]^{2 / 3} \text { and } G^{e}=\frac{(15-12 v) B^{e}}{10-5 v}
$$

In Eq. $1, P^{e}$ denotes the elastic normal force contribution to the average stress, given by [7]

$$
P^{e}=P+\frac{z \Phi F_{0}}{\pi d^{2}} .
$$

Ratios $B / B^{e}$ and $G / G^{e}$, anomalously small in loose states, grow with $P^{*}$, and finally approach values typical of lowcoordination cohesionless grain packs [11]. 


\section{Triaxial compression}

\subsection{Macroscopic behaviour}

Trixial compression tests are applied to several states at different $P^{*}$ values, from $10^{-1}$ to large ones. Variations of deviator $q$ (normalised by initial pressure $P_{0}$ ) and solid fraction $\Phi$, versus axial strain $\epsilon_{1}$ are shown in Fig. 5. The
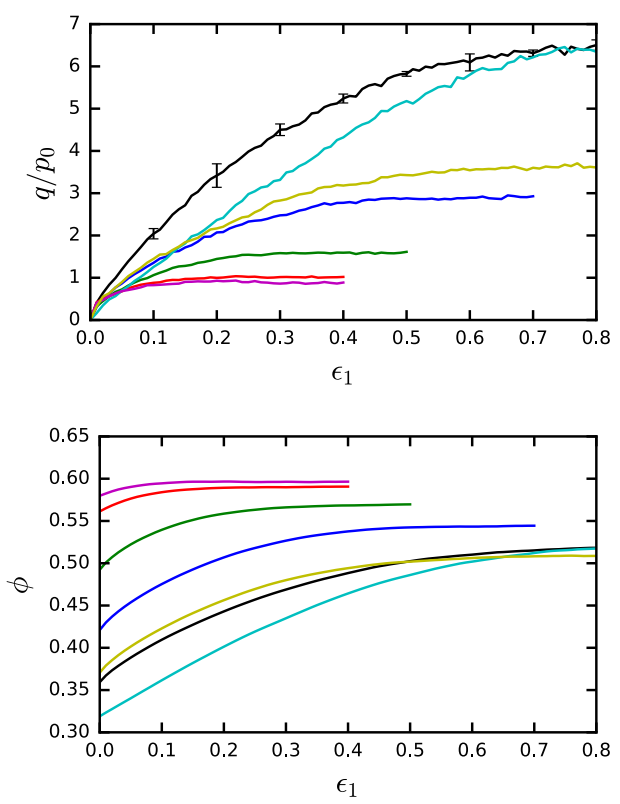

Figure 5. Upper graph: normalised deviator stress $q / p_{0}$, versus axial strain. From top to bottom: $P^{*}=0.1$ (black); $P^{*}=0.1$, but (only in that case) large initial coordination number (light blue); $P^{*}=0.1$, without distant interactions, $D_{R}=0$ (yellow); $P^{*}=0.316$ (dark blue); $P^{*}=1$ (green); $P^{*}=6.32$ (red); $P^{*}=\infty$, i.e. no cohesion (purple). Error bars extend from the lowest to the highest value obtained in 3 samples. Lower graph: solid fraction $\Phi$ vs. $\epsilon_{1}$, same colour code.

test is carried out on controlling strain rate $\dot{\epsilon}_{1}$, such that the inertial number $I=\dot{\epsilon}_{1} \sqrt{m / d P}$ be equal to $10^{-4}$. It is pursued until all measured values approach the plateau corresponding to the critical state, at strains $\epsilon_{1}$ which increase as $P^{*}$ decreases, from $\sim 0.35$ in cohesionless systems up to about 0.8 at $P^{*}=0.1$. The effect of the initial coordination number (and density heterogeneities, see Figs. 2 and 3) only vanishes after this large strain interval. Interestingly, distant attractive forces have quite a notable influence on the critical shear resistance [12], but hardly affect the critical density. We did not record any strain localization, in contrast with the observation of shear bands at $P^{*}=0.1$ in enduring quasistatic shear flow [7].

\subsection{Critical state and yield criterion}

The ratio of deviator stress $q$ to average stress $p=\left(\sigma_{1}+\right.$ $\left.2 \sigma_{3}\right) / 3=P_{0}+q / 3$ would vary linearly with $1 / p$ in a simple Mohr-Coulomb model of plastic yielding at the critical state, with cohesion $c$ and internal friction angle $\varphi$ :

$$
\frac{q}{p}=\frac{6}{3-\sin \varphi}+\frac{6 c \cos \varphi}{(3-\sin \varphi) p}
$$

Fig. 6 shows (as for simple shear tests $[7,8]$ ) a sublinear

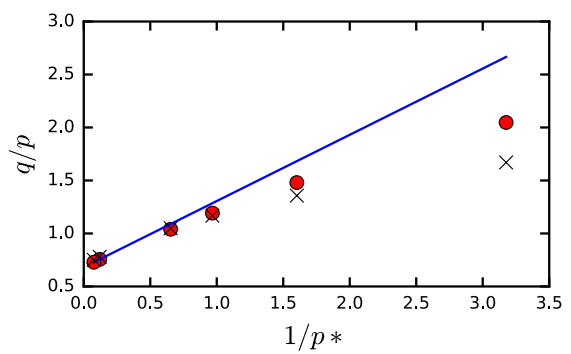

Figure 6. Ratio $q / p$ versus $1 / p^{*}$ at critical state. Straight line: Mohr-coulomb fit to large $p^{*}$ data, using $\varphi$ value of cohesionless case. Red dots: numerical results. Crosses: predictions of Eq. 4.

growth of $q / p$ with $\frac{1}{p^{*}}=\frac{F_{0}}{d^{2}\left(P_{0}+q / 3\right)}$, which is overestimated by the Mohr-Coulomb form identified at large $p^{*}$. Yet a rough estimate of $\frac{q}{p}$ is obtained $[7,8]$ on assuming a $P^{*}$-independent ratio of $q$ to the "effective" average stress due to repulsive elastic forces, introduced as $P_{e}$ in Eq. 2:

$$
\frac{q}{P_{e}}=\frac{6}{3-\sin \varphi}=\left(\frac{q}{p}\right)_{P^{*} \rightarrow \infty} .
$$

Fig. 6 shows that this prediction, although not very accurate for $p^{*}<1$, captures the correct trend.

\subsection{Force networks}

Coordination numbers $z_{c}$ and $z_{d}$ are shown in Fig. 7. Both approach a critical state plateau. The plateau value of $z_{c}$ exhibits little dependence on $P^{*}$, despite the difference in densities, with however a somewhat lower value in the absence of distant attraction. $z_{d}$, on the other hand, increases by a larger amount in denser systems under larger $P^{*}$ (initial pressure and constant lateral stress). But the mechanical influence of those distant forces then decreases as $1 / P^{*}$, and the contribution of distant forces to the deviatoric stress is maximal (about 10\%) among simulated tests for $P^{*}=0.1$ at large strain, down to a few percents for $P^{*} \geq 1$. The major contribution (at least $75 \%$ ) is given by repulsive elastic forces, with tangential forces supplying typically $20 \%$. The influence of distant forces on the critical value of the deviator stress, as apparent in Fig. 5, is mostly indirect, probably due to the stabilization of contact networks: without distant interactions, $z_{c}$ decreases to the smaller value of cohesionless systems.

Although we do not detail this approach here, it may be shown that the deviatoric stress is simply related to anisotropy parameters for fabric tensors and force intensities, as often pointed out for a variety of granular systems [8, 13]. In this respect, the specific feature of the loose systems studied here for smaller $P^{*}$ values is the larger contribution of the anisotropy of forces compared to fabric terms: the contacts oriented near the major principal stress direction tend to carry larger forces, but they are not very much more numerous than those oriented differently. This might be attributed to the structures shown 

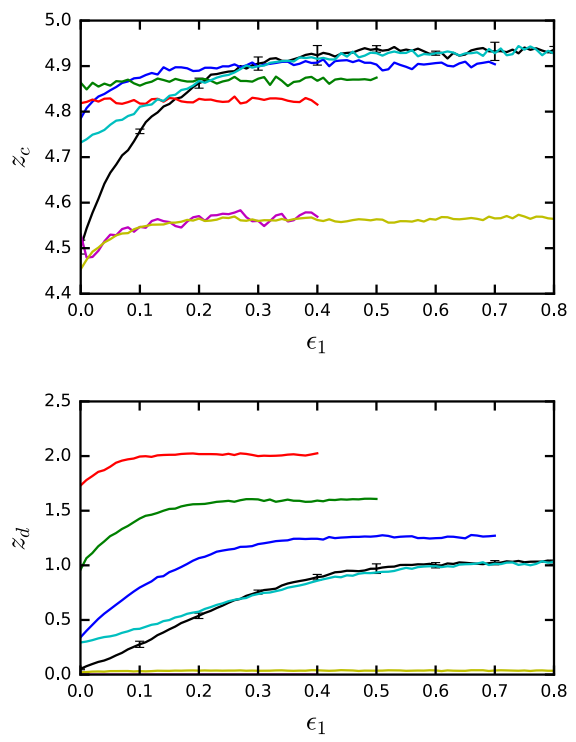

Figure 7. Contact coordination (top graph) and distant interaction coordination number (bottom) versus axial strain. Same colour code as in Fig. 5.

in Fig. 2 likely inducing correlated displacements determined by the mesoscale heterogeneities.

Elastic moduli $[14,15]$ enable a probe of contact network anisotropy. In Fig. 8 the ratio of longitudinal moduli

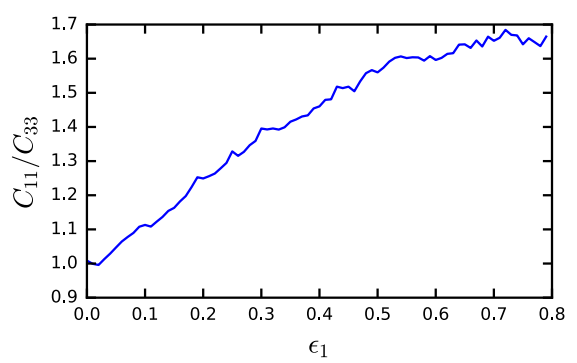

Figure 8. Ratio $C_{11} / C_{33}$ of axial to transverse longitudinal elastic moduli, versus $\epsilon_{1}$ in triaxial compression $\left(P^{*}=0.1\right)$.

(those determining the velocity of longitudinal waves) in the axial and lateral directions is plotted versus $\epsilon_{1}$. Relations of elastic moduli to stress and fabric anisotropies could be exploited in experiments on such systems.

\section{Discussion}

Compared to cohesionless ones, cohesive granular assemblies exhibit a wider variety of structures and properties, and the present communication contributed to the investigation of the little known open, loose systems, with lower reduced pressure $P^{*}$ than in most published studies [12]. As $P^{*}$ increases, irreversible compaction takes place. In triaxial compression, the route to the critical state is longer, involving important density increases. The deviator to mean stress ratio achieves large values, as roughly predicted by an "effective stress" approach. We showed the enduring effect (i.e., up to large strains) of the initial geometry (connectivity and pore size distribution, as determined by initial aggregation process) for the same density, in isotropic or triaxial compression.

The preliminary results presented here should be further completed and analysed in many ways. The slow plastic strengthening and density increase in triaxial compression might be compared to the observed plastic response of gel networks, although those are usually probed at constant volume rather than constant normal (lateral) stress and often modeled differently (e.g., with bonds and angular elasticity [16]). Elastic properties of tenuous networks, and their rupture properties call for detailed investigations. Rolling resistance in contacts [10], which strongly affects loose cohesive structures, and plays an important in colloidal aggregates [17], should be introduced.

Most importantly, the mesoscale density heterogeneities of loose aggregated structures should be related to their mechanical properties.

\section{References}

[1] F. Radjaï, F. Dubois, eds., Discrete-element modeling of granular materials (Wiley, 2011)

[2] B. Andreotti, Y. Forterre, O. Pouliquen, Granular Media: Between Fluid and Solid (Cambridge University Press, Cambridge, UK, 2013)

[3] J. Mitchell, K. Soga, Fundamentals of Soil Behavior (Wiley, 2005)

[4] V.D. Than, S. Khamseh, A.M. Tang, J.M. Pereira, F. Chevoir, J.N. Roux, ASCE J. Eng. Mech. 143, C4016001 (2017)

[5] D. Kadau, G. Bartels, L. Brendel, D.E. Wolf, Phase Trans. 76, 315 (2003)

[6] F.A. Gilabert, J.N. Roux, A. Castellanos, Phys. Rev. E 75, 011303 (2007)

[7] S. Khamseh, J.N. Roux, F. Chevoir, Phys. Rev. E 92 , 022201 (2015)

[8] M. Badetti, A. Fall, F. Chevoir, J.N. Roux, Europ. Phys. J. E 41, 68 (2018)

[9] M. Badetti, A. Fall, D. Hautemayou, S. Rodts, J.N. Roux, J. Rheol. 62, 1175 (2018)

[10] F.A. Gilabert, J.N. Roux, A. Castellanos, Phys. Rev. E 78, 031305 (2008)

[11] I. Agnolin, J.N. Roux, Phys. Rev. E 76, 061304 (2007)

[12] J.P. Wang, G.H. Zeng, H.S. Yu, Comp. Part. Mech. 6, 637 (2019)

[13] E. Azéma, F. Radjaï, F. Dubois, Phys. Rev. E 87, 062223 (2013)

[14] L. La Ragione, V. Magnanimo, Phys. Rev. E 85, 031304 (2012)

[15] M.H. Khalili, J.N. Roux, J.M. Pereira, S. Brisard, M. Bornert, Phys. Rev. E 95, 032908 (2017)

[16] M. Bouzid, E. Del Gado, Langmuir 34, 773 (2018)

[17] J.P. Pantina, E.M. Furst, Phys. Rev. Lett. 94, 138301 (2005) 